\title{
Influence of granulometry in the Hurst exponent of air liquid interfaces formed during capillary rising in a granular media
}

\author{
Guilherme L. Gontijo $^{1, \star}$, Flávia B. Souza ${ }^{1}$, Rafael M. L. Braga ${ }^{2}$, Pedro H. E. Silva ${ }^{2}$, Maury D. Correia $^{5, \star \star}$, and A. P.

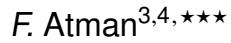 \\ ${ }^{1}$ Civil Engineering Department, Centro Federal de Educação Tecnológica de Minas Gerais, CEFET-MG, Av. Amazonas 7675, \\ Belo Horizonte, MG, Brazil. \\ ${ }^{2}$ Materials Engineering Department, CEFET-MG, Av. Amazonas 5253, Belo Horizonte, Brazil, \\ ${ }^{3}$ Departamento de Física e Matemática, CEFET-MG, Av. Amazonas 7675, Belo Horizonte, MG, Brazil. \\ ${ }^{4}$ National Institute of Science and Technology for Complex Systems, INCT-SC - CEFET-MG. \\ ${ }^{5}$ Centro Brasileiro de Pesquisas Físicas, CBPF. R. Xavier Sigaud, 150, Rio de Janeiro, RJ, Brazil.
}

\begin{abstract}
We report results concerning the fractal dimension of a air/fluid interface formed during the capillary rising of a fluid into a dense granular media. The system consists in a modified Hele-Shaw cell filled with grains at different granulometries and confined in a narrow gap between the glass plates. The system is then placed onto a water reservoir, and the liquid penetrates the medium due to capillary forces. We measure the Hurst exponent of the liquid/air interface with help of image processing, and follow the temporal evolution of the profiles. We observe that the Hurst exponent can be related with the granulometry, but the range of values are odd to the predicted values from models or theory.
\end{abstract}

\section{Introduction}

Fluid flow in heterogeneous media is a wide spreading phenomenon found in several natural and industrial processes, such as groundwater formation, percolation, oil extraction, chromatography, crack formation, etc. [1-3]. Due this feature, researchers from various fields, such as chemistry, physics and engineering in general, became interested on it, and several approaches combining theory, experiment and numerical modeling have been applied [46].

A key concept to understand this phenomena is pattern formation, particularly fractal geometry and analysis [4], and despite several efforts devoted to understand the fluid flow in granular media, a unified description for this phenomena still lacking [7-11]. Previously, Atman et al. have studied the air/liquid interface formed during the capillary rising of water into a granular media [12], but the results did not fit to the expected theoretic models. Here, we present an extension of that work presenting new results for the Hurst exponent of the air/liquid interface now considering granular media with three different granulometries. We also considered different widths for the gap between the plates of the Hele-Shaw cell used in the experiments, and compare the results with previous experiments, simulations and the predicted values of the Deppining by Directed Percolation model (DPD) [4, 5].

\footnotetext{
^e-mail: glgontijo@gmail.com

$\star \star$ e-mail: maury@cbpf.br

$\star \star \star$ e-mail: atman@dppg.cefetmg.br
}

The paper is structured as follows: after this brief introduction, we present the experimental setup and methodology in the next section. After, we discuss the results obtained and close with our conclusions and perspectives.

\section{Experimental Setup}

We consider a Hele-Shaw cell filled with a granular material (sand) with different gradings (granulometries), assembled to form an apparatus which can be placed over an water reservoir - Figure 1. The glass plates have dimensions $(400 \times 300 \times 4) m m$, put apart by spacers with 4 $\mathrm{mm}$ each, placed at the four corners in the gap between the plates. We also run experiments with 2 and 3 spacers for a given grading. To fill the apparatus with sand, a special bond tape is placed at lateral borders to block the water passage, and also help to hold the plates together. The gap at bottom of the apparatus is filled with a fine polystyrene foam to avoid sand evasion but allowing the water passage. The granular media used was a white quartz sand, with different gradings: $45-90 \mathrm{~mm}, 70-120 \mathrm{~mm}$ and $125-600 \mathrm{~mm}$, which means that the grains have $95 \%$ of their average radii within that range. Grain geometries are very different, mostly consisting of polyhedrons with several faces, but which could be approximated by a sphere with a mean radius $r$.

The cell is filled with sand, gently poured atop the cell, with help of a sheet of paper. The top side of the cell is left open and the sand is poured until the gap is completely 

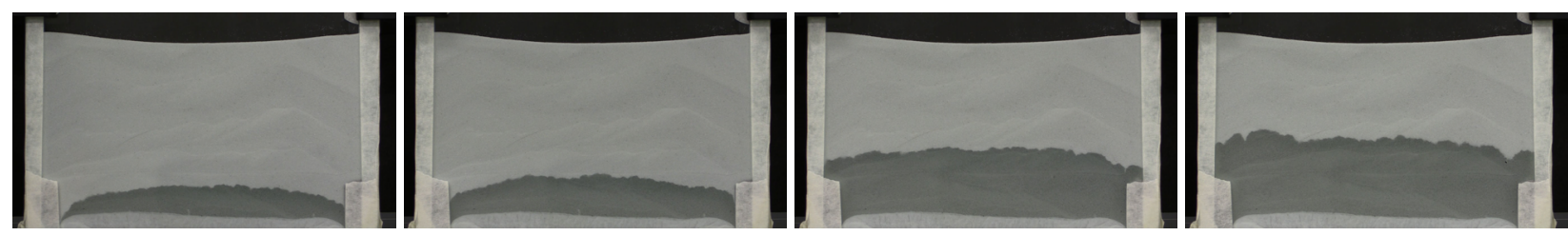

Figure 1. Fluid displacement in heterogeneous media. From left to right, snapshots of the experimental assembly showing the capillary rising of the fluid into the granular media, evincing the high contrast between the wet and dry phases.

filled. During this process, sometimes we tapped the system to compact and homogenize the media, but some degree of segregation is unavoidable. The granular media formed is very heterogeneous, characterized as a highly connected porous media with strong capillary pressure.

Next, the apparatus is fixed at the four corners with clamps, and placed vertically over a water reservoir, partially filled at that moment. A digital video camcorder Canon $^{T M}$ Full HD Vixia HF G30, with (1920x1080) pixels, was placed at $1.5 \mathrm{~m}$ in front of the assembly. The level of water in reservoir is then slowly raised until it forms a flat interface with the glass bottom. Just before the level of the water in the reservoir reaches the bottom of the plates, the camera is turned on. A complete record of an experiment typically takes around 15 minutes, thus we can disregard evaporation effects and consider the water-glass interface angle as constant - the volume of water of the reservoir $(40 L)$ is much bigger than the water volume inside the cell $(100 \mathrm{~mL})$.

As soon the water touch the granular media, a liquid/air interfaced is formed, altering the contrast of wet area, allowing the identification of the interface after image treatment. The images were treated with Image ${ }^{T M}$ software with a sequence of numerical operations [6], as detailed in Figure 2. As resutl, a single valued function corresponding to the profile of the liquid/air interface, $h(x . t)$, where $x$ indicates the horizontal position, can be extracted.

We have performed five runs considering exactly the same procedure described above, for each set of the tested parameters. For each run, we and took snapshots of the cell along all the experiment, at predetermined times. We calculate the Hurst exponent $H$, for each interface considered, using detrended fluctuation analysis (DFA) [13, 14]. Then, the average value of the Hurst exponents at a given time, $H(t)$, can be plotted against time - Figure 3 .

The roughness of the interface is defined as the variance of the function $h(x, t)$ :

$$
w(t)=\sqrt{\frac{1}{2 N} \sum_{i}^{N}\left(h_{i}-\bar{h}\right)^{2}}
$$

where $h_{i}$ is the height of the interface at the column $i$, and $i$ is numbered in function of the pixel resolution of the camera. DFA considers the roughness in a local scale $\epsilon$ regarding to the best linear fit for the data in this scale:

$$
w(\epsilon)=\sqrt{\frac{1}{1+2 \epsilon} \sum_{i=\epsilon}^{i+\epsilon}\left(h_{i}-\left(\alpha(\epsilon) x_{i}+\beta(\epsilon)\right)\right)^{2}},
$$


Figure 2. Image treatment of the air/liquid interface. The top panel shows the image captured from the digital camera, which was treated with Image ${ }^{T M}$ in order to obtain a single valued function, shown above the treated image. The bottom panel show the methodology to calculate the Hurst exponent, eq. 3. The continuous line indicates a power law fit giving the exponent $H=0.797(7)$.

where $\alpha(\epsilon)$ and $\beta(\epsilon)$ are, respectively, the angular and the linear fit coefficients at the local scale $\epsilon$.

Then, we can obtain the Hurst exponent from the relation:

$$
w(\epsilon) \sim \epsilon^{H},
$$

as shown in Figure 2. In a $\log \times \log$ plot, it corresponds to the angular coefficient of the curve. The linear behavior of $w(\epsilon)$ is a sign of the self-affinity of the function considered, confirming its fractal feature.

\section{Results and Discussion}

We have performed five runs for each set of experimental parameters, and the results shown correspond to the average values. Figure 3 show the results for different spacers and (45 - 90 grading. We can observe that as the gap width increases, the results become more noisy, and fluctuates considerably. This is consistent with a layerby-layer forming interface, and could be related with the three dimensional feature of the media as the gap width increases. Considering the Hurst exponent, the stationary results shown a tendency for a decreasing of its value as 


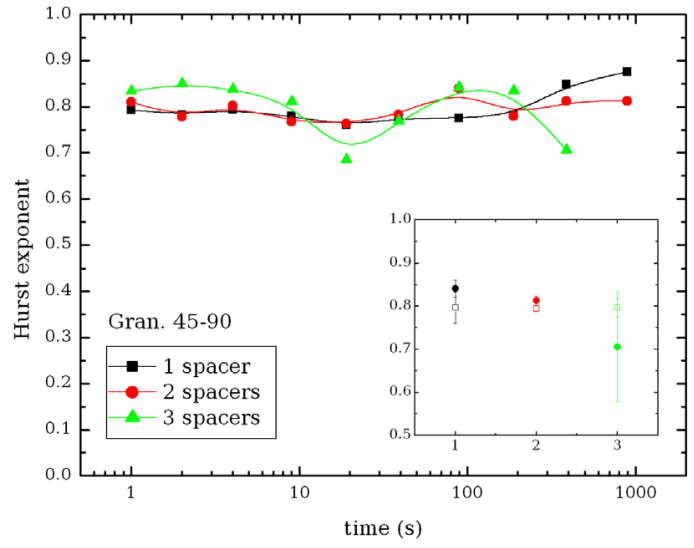

Figure 3. Temporal evolution of the Hurst exponent considering different gap widths. The grading considered was $45-90 \mathrm{~mm}$ and we show results for 1, 2 and 3 spacers placed in the gap. Despite the curves are pretty close to each other, in the inset its possible to verify a tendency on the Hurst exponent value to decrease as the gap width increases. In the inset, open symbols correspond to the temporal average of the Hurst exponent, and the filled ones correspond to stationary values.

the spacing increases, a results which indicates that the interface become more flat with. Again, this result is compatible with the three-dimensional feature of the interface as the gap width increases.

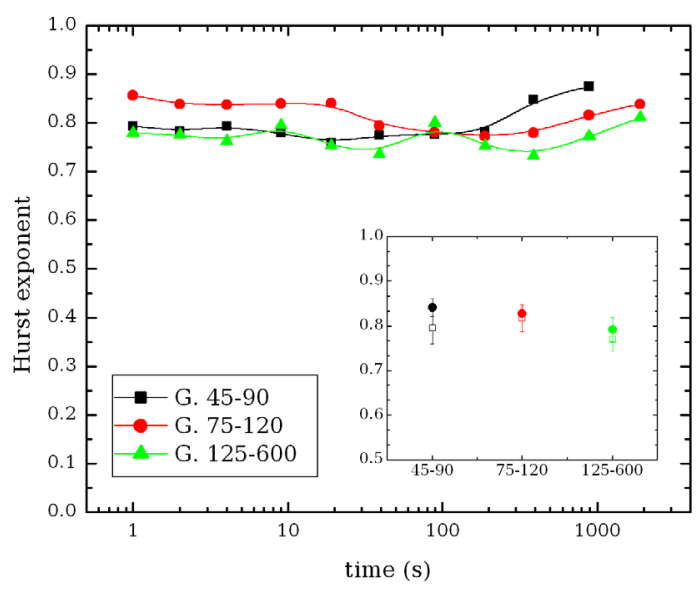

Figure 4. Time evolution of the Hurst exponent for the air/liquid interface for different sand gradings. Again, the curves are quite close to each other, but we can observe a tendency of smaller values of Hurst exponent when the grading is larger, an observation which agrees with our expectation since the media became more connected as the granulometry increases.

In Figure 4 we shown the results for the Hurst exponent obtained for the same gap width (single spacer) but with different gradings. Surprisingly, the results do not shown great dispersion. Only a slight tendency to decreas- ing of the Hurst exponent value was observed for larger values of gradings. All results for the Hurst exponent fluctuates around $H \sim 0.8$. This value do not agree with none model or other experiment that we are aware, except [? ]. The conclusion is that the high connectivity of the granular media implies to smoother surfaces, rising the value of the Hurst exponent above to described in literature. We have developed a model to study this problem, but for the moment our results do not fit well the experiments.

\section{Conclusions and Perspectives}

we have studied the influence of the gap width and the sand grading on the value of the Hurst exponent of the air/liquid interface formed during the displacement of air by water into a heterogeneous granular media. Our results indicates that an average value of $H \sim 0.8$ for all experiments, a result which was quite surprisingly for us. A slight tendency of smoother surfaces (smaller $H$ values) was observed for larger gaps and increasing the grading. We hope to extend this work using grading ranges more narrow and also testing thicker and narrower gap widths.

\section{Acknowledgements}

PHES and FBS thank CNPq for financial support. APFA and RMB thank CEFET and FAPEMIG for financial support.

\section{References}

[1] Y. Shikhmurzaev, Capillary Flows With Forming Interfaces (Chapman \& Hall/CRC, 2008), ISBN 9781584887485

[2] M. Sahimi, Review Modern Physics 65, 1393 (1993)

[3] D. Dalmas, E. Barthel, D. Vandembroucq, Journal of the Mechanics and Physics of Solids 57, 446 (2009)

[4] A.L. Barabási, H.E. Stanley, Fractal Concepts in Surface Growth, 1st edn. (Cambridge University Press, 1995)

[5] L.A.N. Amaral, A.L. Barabási, S.V. Buldyrev, H.A. Makse, H.E. Stanley, Physical Review E 52, 4087 (1995)

[6] A. Atman, Crescimento de interfaces em meio poroso (1998), also available at http://www13.fisica.ufmg.br/ posgrad/ (in Portuguese)

[7] P.B. Umbanhowar, F. Melo, H.L. Swinney, Nature 382, 793 (1996)

[8] S.F. Pinto, M.S. Couto, A.P.F. Atman, S.G. Alves, A.T. Bernardes, H.F.V. de Resende, E.C. Souza, Physical Review Letters 99 (2007)

[9] C. Chevalier, A. Lindner, E. Clément, Physical Review Letters 99 (2007)

[10] A. Lindner, D. Bonn, E.C. Poiré, M.B. Amar, J. Meunier, Journal of Fluid Mechanics 469, 237 (2002)

[11] J. Nase, A. Lindner, C. Creton, Physical review letters 101, 074503 (2008) 
[12] A. Atman, G. Combe, T. Ferreira, J. Barros, Pattern formation during capillary rising of a fluid front into a granular media, in POWDERS AND GRAINS 2013: Proceedings of the 7th international conference on micromechanics of granular media, AIP Conference Proceedings (2013), Vol. 1542, pp. 1075-1078
[13] C.K. Peng, S.V. Buldyrev, S. Havlin, M. Simons, H.E. Stanley, A.L. Goldberger, Physical Review E 49, 1685 (1994)

[14] J.G. Moreira, J.K.L. da Silva, S.O. Kamphorst, Journal of Physics A: Mathematical and General 27, 8079 (1994) 